\title{
Creating Shared Value: Eine Grundsatzkritik
}

\section{THOMAS BESCHORNER UND THOMAS HAJDUK ${ }^{*}$}

Der Beitrag formuliert eine Grundsatzkritik an Michael Porters und Marc Kramers Konzept des „Creating Shared Value“ (CSV). Für diesen Zweck wird in einem ersten Schritt die positive und negative Kritik, die seit 2011 am CSV geäußert worden ist, zusammengefasst. Im Weiteren zeigen die Autoren, dass CSV hinter das Verständnis einer modernen Unternehmensverantwortung zurückfällt, die insbesondere in einer angemesseneren Verhältnisbestimmung von Unternehmen und Gesellschaft liegen muss. Der Beitrag schließt mit kritischen Anmerkungen zur Rolle der Wissenschaft in der Interaktion mit der Praxis.

Schlagwörter: Creating Shared Value, Corporate Social Responsibility, Unternehmensethik, Kritik

\section{Creating Shared Value: A Fundamental Critique}

This article offers a fundamental critique of Michael Porter's and Marc Kramer's "Creating Shared Value" (CSV) concept. First, the authors summarise the positive and negative criticism which CSV has received since 2011. They then show that CSV falls short of a modern understanding of corporate responsibility which is centred on more adequate ideas about the relationship between business and society. The article concludes with critical comments on the role of scholars in their interaction with practitioners.

Keywords: Creating Shared Value, Corporate Social Responsibility, Business Ethics, Critique

\section{Einleitung}

„Creating Shared Value“ (CSV) - gemeinsame Werte schaffen - so lautet der Vorschlag der Harvard-Professoren Michael Porter und Mark Kramer (2011). In einem vielbeachteten Artikel der Harvard Business Review empfehlen sie ein stärker wertebasiertes Management, das zu nicht weniger als einer Neuerfindung des Kapitalismus führen soll. An die Stelle eines verengten Managementverständnisses mit seiner Fixierung auf kurzfristigen Profiten, so die Hauptthese der Autoren, soll die Schaffung gemeinsamer Werte rücken. Damit soll es gelingen, das gegenüber der Wirtschaft

\footnotetext{
Prof. Dr. Thomas Beschorner, Institut für Wirtschaftsethik, Universität St.Gallen, Girtannerstrasse 8, CH-9010 St.Gallen, Tel.: +41-(0)71 224 3143, Fax: +41-(0)71 224 2881, E-Mail: thomas.beschorner@unisg.ch, Forschungsschwerpunkte: Wirtschafts- und Unternehmensethik, Handlungs- und Institutionentheorie.

Thomas Hajduk, Institut für Wirtschaftsethik, Universität St.Gallen, Girtannerstrasse 8, CH9010 St.Gallen, Tel.: +41-(0)71 224 3105, Fax: +41-(0)71 224 2881, E-Mail: thomas. hajduk@unisg.ch, Forschungsschwerpunkte: Internationale CSR-Standards und Global Governance, Geschichte der Unternehmensverantwortung.
} 
verlorene Vertrauen zurückzugewinnen. Es geht Porter und Kramer um eine Reintegration und eine Wiedereinbettung der Wirtschaft in die Gesellschaft.

Creating Shared Value ist in den vergangenen Jahren zu einem beachteten „Buzzword" in der Unternehmenspraxis ebenso wie in Teilen der Managementlehre geworden. Die Prominenz des Hauptautors Michael Porter und die positiv konnotierte Begriffskomposition scheinen Anklang bei einigen Zielgruppen zu finden. Mit etwa 1.900 Zitationen bei Google Scholar innerhalb von knapp vier Jahren kann der Beitrag als äußerst einflussreich eingestuft werden. Auch wenn unseres Wissens keine detaillierten Daten zur Rezeption des Beitrages vorliegen, liegt man sicherlich nicht falsch, wenn man die Reaktionen darauf als weitestgehend positiv und zustimmend annimmt; auch die anderen Beiträge in dieser Ausgabe der zfwu zeugen von einer tendenziell positiven Rezeption.

Das bedeutet jedoch nicht, dass der Ansatz völlig kritiklos angenommen wurde. Der Economist (2011) titelte schon früh „Oh, Mr Porter“ und konstatierte: „the new big idea from business's greatest living guru seems a bit undercooked" - und das ist, wie wir sehen werden, noch eine untertriebene Einschätzung gegenüber CSV. Weitere und weiter gehende kritische (wissenschaftliche) Betrachtungen des vorgeschlagenen Konzeptes blieben aber erst einmal nahezu aus, was uns sehr bemerkenswert erscheint. In den Jahren 2013 und 2014 sollten dann jedoch eine Reihe - nahezu parallel erscheinender - wissenschaftlicher Artikel (vgl. Beschorner 2013a; Hartman/Werhane 2013; Crane et al. 2014) - veröffentlicht werden, die CSV mitunter harsch kritisieren.

Wir wollen diese kritischen Positionen im Folgenden nicht en detail nachzeichnen, sondern uns bei unserer Darstellung lediglich auf einige ausgewählte Aspekte der Diskussion konzentrieren und diese knapp umreißen. Auf der Grundlage dieser Kritik wollen wir dann in einem zweiten Schritt andeuten, was - im Gegensatz zu CSV moderne Unternehmensverantwortung auszeichnet. Wir enden mit einigen Reflexionen zum Verhältnis von Unternehmen und Gesellschaft einerseits sowie der Rolle von Wissenschaft in diesem Zusammenhang andererseits.

\section{CSV: positive und negative Kritik}

Mit Creating Shared Value soll der Kapitalismus neu erfunden werden und eine Innovations- und Wachstumswelle ausgelöst werden, von der sowohl Unternehmen als auch die Gesellschaft profitieren. Wir wollen fragen: Inwieweit tragen die Vorschläge der Autoren? Läuten die beiden Autoren eine Trendwende ein, in deren Folge Corporate Social Responsibility (CSR) durch Creating Shared Value ersetzt wird?

Tatsächlich deutet das CSV-Konzept in Teilen einige fortschrittliche Ideen an: Interessant ist beispielsweise, dass die Autoren die möglichen positiven Beiträge von Unternehmen betonen und diese in den Mittelpunkt des Interesses rücken. Sie lehnen damit ein bloß (Reputations-)Risiken vermeidendes, defensives Konzept ab. Unternehmen sollten stattdessen ihre Fertigkeiten, Ressourcen und Managementfähigkeiten nutzen, um sozialen Fortschritt anzuführen (vgl. Porter/Kramer 2011: 77).

Auch wenn dieses Verständnis von Unternehmen als proaktive Akteure in der akademischen Diskussion bereits seit einiger Zeit umfangreich behandelt wird (vgl. Zadek 2001; Beschorner 2004; Matten/Crane 2005; Palazzo/Scherer 2007), so weist diese 
Ausrichtung durchaus in eine richtige und wichtige Richtung. Damit zusammenhängend und darüber hinausgehend schlagen Porter und Kramer vor, von gesellschaftlichen „Bedürfnissen“ her - statt in den sonst üblichen Kategorien „Produkte“ und „Dienstleistungen“ - zu denken und diese als Ausgangspunkt für Innovationen zu wählen. Diese Unterscheidung ist sowohl theoretisch als auch praktisch von großer Bedeutung für unternehmerische Geschäftsmodelle: Geht man etwa von einem Bedürfnis nach Mobilität aus, so wären die Herstellung und der Verkauf von Kraftfahrzeugen ein mögliches Mittel zum Zweck. Darüber hinaus lassen sich aber andere Produkte und Leistungen denken, um Menschen mit Mobilität zu versorgen: Aus Autobauern würden so Mobilitätsunternehmen, die neue, nachhaltige Märkte erschließen (z.B. Car Sharing, Mobilitätspässe, etc.).

Können Porter und Kramer mit diesen zumindest angedeuteten Ideen noch positiv überraschen, zeigt sich in der ökonomischen Argumentation der Autoren die orthodoxe Prägung ihrer Zunft. Nicht zufällig haben sie den Beitrag im Harvard Business Review veröffentlicht und auf die Leserschaft aus Betriebswirten und Managern ausgerichtet. Früh machen sie klar, dass CSV weder mit sperrigen normativen Begriffen wie Gerechtigkeit, Verantwortung oder Gemeinwohl noch mit den bekannten CSRDebatten verbunden ist, sondern es um ein neues Verständnis von Wertschöpfung geht. Mit diesem begrifflichen Wandel von „Verantwortung“ (Responsibility) zu „Wertschöpfung" (Value) soll es gelingen, einen bis dato skeptischen Mainstream anzusprechen, der sonst weiterhin auf Distanz bliebe, meinen Porter und Kramer (2014: 150). Die von ihrer Unternehmensberatung Foundation Strategy Group gestartete „Shared Value Initiative“ lädt Unternehmen, Zivilgesellschaft und Politik ein, das Konzept in der Praxis umzusetzen.

Der Versuch, CSR-Skeptiker für eine verantwortungsvolle Unternehmensführung zu sensibilisieren und zu gewinnen, erweist sich bei genauerer Betrachtung jedoch als hoch problematisch. Porter und Kramer bringen nämlich ihren CSV-Ansatz gegen einen längst überholten CSR-Begriff in Stellung, in dem sie CSR als rein philanthropische Tätigkeiten beschreiben, die vom Kerngeschäft des Unternehmens losgelöst sind, während CSV in das Kerngeschäft integriert sei (vgl. Porter/Kramer 2011: 76). Ein solch simples CSR-Verständnis vertritt heute aber so gut wie niemand mehr, weder in der Theorie noch der Praxis (vgl. Beschorner 2013a). Bei aller definitorischen Vielfalt im Feld besteht dennoch Konsens darüber, dass CSR kein Appendix zu unternehmerischen Tätigkeiten, sondern zentraler Bestandteil des Kerngeschäfts ist. In der Tat: Bei CSR geht es nicht darum, wie Profite ausgegeben, sondern wie sie erwirtschaftet werden - was sich auch bis nach Boston hätte herumsprechen können.

Porter und Kramer kreieren hier Corporate Social Responsibility stattdessen als „Pappkameraden“ (vgl. Beschorner 2013a: 109) zur Plausibilisierung ihrer „big idea“. Crane et al. (2014: 134) mahnen die eigentümliche Verwendung von CSR durch die Autoren in ihrer Kritik ebenso an, müssen dann aber feststellen, dass Porter und Kramer (2014) Erläuterungen dazu in ihrer Replik offensichtlich für nicht notwendig erachten. Um es mit aller Deutlichkeit zu sagen: Es handelt sich bei dieser Vorgehensweise aus unserer Sicht um ein wissenschaftlich unredliches Vorgehen, das man bei Bachelorarbeiten mit Notenabzug ahnden müsste. Mit ihrer Kritik an CSR demonstrieren Porter und Kramer, wie begrenzt das Verständnis orthodoxer Betriebs- 
wirte von Unternehmensverantwortung noch immer ist. Hier hat sich seit Milton Friedmans berühmter Kritik von 1970, wonach CSR nichts weiter als Philanthropie auf Kosten der Shareholder sei, scheinbar nicht viel getan (vgl. Friedman 1970; zur Historizität des Beitrags: Hajduk 2015).

Auch in ihrer sonstigen Argumentation bleiben die Autoren in ihrem neoklassischen Paradigma gefangen. So ist die eindimensionale Profitmaximierung, die sie mit CSV eigentlich überwinden wollten, der normative Dreh- und Angelpunkt ihres Ansatzes. Profitmaximierung wird nicht nur empirisch vorausgesetzt - Unternehmen seien im Wettbewerb dazu gezwungen - sondern als moralisch gut angenommen. CSV bekräftigt diese normative Festlegung, indem Unternehmen weiterhin Profite machen und dabei „gesellschaftliche Bedürfnisse“ der Gesellschaft abdecken. Entscheidend bleibt aber das reine Profitmotiv; Bedürfnisse dienen lediglich als Mittel zum Zweck, indem Unternehmen einen Absatzmarkt erkennen, den Business Case kalkulieren und gewinnbringend investieren. Solche Unternehmen wären gute „Rechner“ im Sinne eines Homo oeconomicus; mit verantwortungsvollem, ethischem Verhalten hätte das aber wenig zu tun. ${ }^{1}$

Selbst in solchen Fällen, in denen ein „gemeinsamer Wert“ für Unternehmen und Gesellschaft geschaffen wird, also in so genannten „A-Cases“ (vgl. Scholz 2014), lässt CSV weder Neues erkennen noch kann es sich von seiner ökonomischen Perspektive lösen. Crane et al. (2014: 143) und Beschorner (2013a: 110) erinnern daran, dass es bereits seit längerem Ansätze in der Managementtheorie gibt, die in Unternehmen mehr als rein ökonomische Akteure sehen, und führen zuvörderst die StakeholderTheorie an. Letztere sei vor allem deswegen so erfolgreich gewesen, weil zwei ihrer zentralen Annahmen - Unternehmen werden von der Gesellschaft beeinflusst, weswegen gutes Management mit gesellschaftlichen Gruppen interagiere - kompatibel mit einer unternehmenszentrierten, ökonomischen Sichtweise seien. CSV schlage in dieselbe Kerbe, weil dadurch gesellschaftliche Ansprüche auf rein ökonomische Weise beantwortet werden. Dies führt nebenbei dazu, dass CSV eine Schwäche des Stakeholderansatzes teilt (vgl. Beschorner 2013a: 110): Unternehmen stellen nur „relevante“ - und das sind in erster Linie einflussreiche - Stakeholder zufrieden, ohne aber die ethisch möglicherweise ebenfalls gerechtfertigten Forderungen der „claimholders“ (Waxenberger/Spence 2003) zu berücksichtigen und damit auf eine tatsächliche gesellschaftliche Legitimität abzustellen.

Die ökonomische Logik der „A-Cases“ stößt vollends an ihre Grenzen, wenn ein Unternehmen keinen Mehrwert aus der Auseinandersetzung mit der Gesellschaft generieren kann bzw. wenn unternehmerische und gesellschaftliche Interessen nicht Hand in Hand gehen, sondern vielleicht einander entgegenstehen. In diesen „BCases" greift CSV nicht, wie Scholz (2014) deutlich macht (vgl. dazu auch den Beitrag von Scholz und de los Reyes in diesem Heft). Stattdessen verweisen Porter und Kramer (2011: 76) auf die Einhaltung von „Recht und ethischen Standards“, ohne auszuführen, welche Standards sie meinen. Diese neoklassische Vorstellung zweckrational agierender und gewinngetriebener Unternehmen innerhalb eines gesetzlich (freilich

1 Wir haben CSV daher an anderer Stelle als „One-Trick Pony Approach“ charakterisiert; vgl. Beschorner (2013a). 
nicht zu stark) regulierten Rahmens deutet auf die Begrenzung des Unternehmensverständnisses der beiden Autoren.

Es ist richtig, dass Unternehmen gewinnorientierte Akteure sind und in einer ökonomischen Logik agieren. Sie sind aber zugleich auch gesellschaftliche Akteure und verstehen es als solche in verschiedenen Logiken mit ihrer Umwelt zu interagieren (vgl. Matten/Crane 2005). So sind sie etwa nicht bloße Objekte von Recht und Gesetz, sondern nehmen als politische Akteure etwa über Lobbying und Wahlkampfspenden aktiv am politischen Willensbildungsprozess teil oder nehmen im gesellschaftlichen Diskurs einen Standpunkt ein (vgl. Curbach 2009). Unternehmen können sich als „corporate citizens“ in ihrer Region engagieren und so ihre gesellschaftliche Lebenswelt mitgestalten, was sowohl auf Akzeptanz als auch Widerstand der Bürger stoßen kann. In solchen Fällen geht es nicht um einen ökonomischen Mehrwert für das Unternehmen; vielmehr äußern sich Unternehmen in den verschiedenen Rollen, die sie innerhalb der Gesellschaft bekleiden. Unternehmen sprechen also mehrere Sprachen, abhängig von dem Kontext. Wenn Porter und Kramer mit CSV vorgeben, dass Unternehmen und Manager allzeit nur die eine Sprache der Wertschöpfung sprächen, so leisteten sie insbesondere ihren Studenten einen Bärendienst (vgl. Donaldson 2014).

Anstelle des funktionalen CSV-Konzepts brauchen Unternehmen ein ethisches Leitbild, wonach sie sich nicht nur als ökonomische, sondern gesellschaftliche Akteure begreifen. Dabei geht es um Aspekte wie die fundamentalen Unternehmenswerte oder den Beitrag zur Gesellschaft. Ein solches normatives Leitbild bildet die normative Grundlage, auf der Unternehmen verantwortungsvoll handeln können. Dies schließt Profitmaximierung nicht aus. Im Gegenteil: Die normative Grundlage verleiht Profiten erst ihre Legitimität. Diese Legitimität und der damit verbundene (Wieder-) Gewinn von gesellschaftlichem Vertrauen sind auch für die Neuerfindung des Kapitalismus erforderlich, die sich Porter und Kramer auf die Fahnen geschrieben haben. Es reicht dafür nicht, die alte Geschichte von ökonomischer Rationalität, Innovationen und Wachstum zu erzählen. Ein echter Paradigmenwechsel braucht bessere Ideen und mutigere Vorschläge, die dann auch geeignet sein können, Unternehmen wieder als Teil der Gesellschaft zu sehen.

\section{Moderne Unternehmensverantwortung geht anders}

Es sollte deutlich geworden sein: CSV ist kein überzeugender Gegenentwurf zu CSR. Im Gegenteil, es fällt deutlich hinter die sich zunehmend entwickelnde Diskussion zu einer modernen Unternehmensverantwortung zurück. Was aber ist mit einer „,modernen Unternehmensverantwortung“ gemeint? Zunächst einmal bedeutet dies, die vermeintliche Schwäche des CSR-Begriffs - die Offenheit und Vieldeutigkeit - als Stärke $\mathrm{zu}$ sehen. CSR ist die begriffliche Klammer für Diskurs und Praxis von Unternehmensverantwortung. Als solches ist der Begriff wie viele andere Grundbegriffe - etwa „Freiheit", „Demokratie“ oder „Öffentlichkeit" - dem (historischen) Wandel unterzogen und bedarf der dialogischen (Neu-)Bestimmung. Bei CSR geht es also weniger um eine konkrete Definition als vielmehr um die Reflexion verschiedener Vorstellungen von Unternehmensverantwortung sowie der empirisch prüfbaren Unternehmenspraxis. Aus unserer Sicht gehören zu einer so verstandenen CSR u.a. die folgenden Aspekte: 
Es bedarf, erstens, institutioneller Regeln und Strukturen im Unternehmen ebenso wie in der Gesellschaft (vgl. Wieland 1999). In einer freiheitlichen, weltoffenen Gesellschaft stößt die Individualethik, auf die z.B. der ,ehrbare Kaufmann“2 abzielt, an ihre Grenzen. Sie ist zu divers, um eine wie auch immer geartete Ehre zu begründen, noch können vor ihrem Hintergrund prädefinierte Tugenden als uneingeschränkt gültig angenommen werden. Die Individualethik ist daher unbedingt durch eine Institutionenethik zu ergänzen (nicht jedoch zu ersetzen) (vgl. Hajduk/Schank 2015). Diesen Institutionen, beispielsweise in Form eines Verhaltenskodexes oder eines weitergehenden Wertemanagementsystems, kommt dabei eine dreifache Funktion zu:

- Sie entlasteten das Individuum von permanenten moralischen Entscheidungssituationen, die jedes Individuum überfordern würden;

- $\quad$ klar definierte und transparente Regeln schützen vor Willkür von Unternehmenslenkern; und

- $\quad$ sie signalisieren und kommunizieren nach innen und nach außen klar verlässliche Werte (z.B. über eine Unternehmensphilosophie) (vgl. Wieland 1999; Gelbman/Baumgartner 2012; Lorentschitsch/Walker 2012).

Zweitens, rein funktionalistische und werte-entleerte Konzepte, wie bei CSV, sind weder normativ angemessen noch empirisch zutreffend. Wir finden in Unternehmen faktisch vielfältige Werteorientierungen, die die Identität des jeweiligen Unternehmens maßgeblich bestimmen. Unternehmensverantwortung hat hier einerseits eine empirische Aufgabe zur Bestimmung dieser Werteorientierungen, anderseits eine reflexive Aufgabe im Sinne eines kritischen Hinterfragens des Faktischen in konstruktiver Absicht.

Die Umsetzung, das (Vor-)Leben von Werten im unternehmerischen Kontext ist dabei an Fähigkeiten geknüpft, die in der empirischen Analyse und der normativen Reflexion zu berücksichtigen sind:

- Individuelle Fähigkeiten wie etwa (selbst-)kritisches Denken, Verantwortungsgefühl und (fachliches) Wissen.

- Organisationale Fähigkeiten ermöglichen und fördern werteorientiertes Handeln, beispielsweise in Form von langfristigen Vergütungsmodellen, bewusstseinsschaffenden CSR-Programmen, Möglichkeiten Missstände offenlegen zu können (z.B. über „Whistle-Blowing-Systeme“) oder Innovationsprozessen, in die durch CSR soziale und ökologische Meilensteine integriert werden.

- Sprachliche Fähigkeiten zur Kommunikation für einen Dialog mit Anspruchsgruppen sind eine notwendige Voraussetzung zur Schaffung von Legitimität wirtschaftlichen Handelns. Der Begriff der „Verantwortung“ weist auf einen solchen Dialog hin, der über ein strategisches Stakeholdermanagement bzw. die bloße Beachtung wirkmächtiger Stakeholder, wie sie bei CSV gedacht wird (vgl. Beschorner 2013a: 110; Crane et al. 2014: 137), hinausgeht. 
Mit diesem letztgenannten Aspekt gehen, drittens, auch neue Rollen und Verantwortungen für Unternehmen einher. Unternehmen sind hier nicht nur Teil des Produktionssystems, sondern beteiligen sich an einem breiten Spektrum gesellschaftlicher Governance-Prozesse, bei dem es um Verteilung und Verwaltung öffentlicher (globaler) Güter geht (vgl. Wolf/Schwindenhammer 2011). Markttransaktionen und Verhandlung sind ein möglicher Modus dieser Prozesse, der Unternehmen bereits vertraut ist. Es gibt aber auch andere Modi gesellschaftlicher Teilhabe, wie die Teilnahme an gesellschaftlichen Diskursen und deliberativen Prozessen.

Einen zunehmend wichtiger werdenden Bereich stellt in diesem Zusammenhang die (politische) Rolle von Unternehmen als gleichberechtigte Akteure in öffentlichprivaten Partnerschaften und bei ko-regulativen Initiativen dar, die sich immer häufiger in der Praxis beobachten lassen (vgl. Beschorner et al. 2013). Insbesondere das Verhältnis von Unternehmen zu öffentlichen Akteuren verändert sich dadurch und wird komplexer (vgl. Schmiedeknecht/Wieland 2012). Neben der einseitigen Einhaltung von Gesetzen geht es nun mehr auch um gemeinsam getragene und verantwortete Aktivitäten, etwa bei der Schaffung und Implementierung von Soft Law.

\section{Unternehmen und Gesellschaft ...}

Mit diesem letztgenannten Aspekt tangieren wir eine Thematik, die auch Andy Crane und Ko-Autoren (2014) am Ende ihres Artikels andeuten und die uns nicht nur für eine Kritik am CSV-Ansatz wesentlich erscheint, sondern Überlegungen zu einer Unternehmenstheorie und -ethik insgesamt betrifft, nämlich eine angemessene Verhältnisbestimmung von Unternehmen und Gesellschaft, von Gesellschaft und Unternehmen. Für eine derartige Verhältnisbestimmung gilt es unseres Erachtens einerseits ein angemessenes Verständnis von Unternehmen zu entwickeln, wozu weder „as if“Konstrukte noch Theorien einer normativen Ethik geeignet erscheinen. Vielmehr setzt dies empirische Perspektiven voraus, getreu dem Motto „Corporations ARE what they DO“ (Post et al. 2012: 8). Andererseits gilt es ebenso in empirischen Perspektiven ein ungefähres, zwar bearbeitbares, zugleich jedoch auch hinreichend komplexes Bild von gesellschaftlichen Kontexten zu entwickeln. Preston formulierte dazu bereits vor vierzig Jahren:

„serious analysis of the corporation-society relationship requires rigorous and comprehensive conceptions of both the corporation and society; and these conceptions must be articulated in comparable, or at least translatable, terms" (Preston 1975: 446).

Und das erscheint wichtig, so Preston, weil:

„(...) society at large might be interested in performance features of the economic system other than the amount of total current output and its distribution" (Preston 1975: 446).

Wenn wir zu dieser Programmatik noch einmal einen Seitenblick auf die Überlegungen von Porter und Kramer werfen, sind wir, erstens, der Auffassung, dass das zugrunde gelegt Unternehmensverständnis zu reduktionistisch ist (one-trick pony). Zweitens, obwohl ein Bemühen zu verzeichnen ist, sich über Begriffe wie Zivilgesellschaft und Regulierungen gesellschaftstheoretischen Fragen zuzuwenden, zeichnen Porter und 
Kramer auch hier nur ein sehr unzureichendes Bild, das theoretisch unterentwickelt ist. Drittens, setzt CSV hinsichtlich der "translatable terms” (Preston) auf eine strikte Übersetzungsleistung in Nutzenkategorien und verbindet damit zugleich ,a very narrow dated notion of what the corporation is" (Crane et al. 2014: 143). Die Verbindung zwischen Unternehmen und Gesellschaft orientiert sich damit an einer Unternehmensperspektive. Die damit verbundene ökonomische Zentrierung spiegelt sich auch in der zentralen Begrifflichkeit von CSV wider: „Wert(schöpfung)“ bzw. „(creating) value" ist ein ökonomischer Begriff, der andere - nicht ökonomische - unternehmensethische Begriffe wie unternehmerische Verantwortung oder Unternehmensbürger (im Sinne eines citoyen) ersetzen soll.

Crane et al. formulieren zur unternehmenszentrierten Perspektive von Porter und Kramer zutreffend und prägnant:

„to study CSR properly, or for that matter CSV, we cannot start with a notion of what a business is and let its role in society just follow from this" (Crane et al. 2014: 143).

Wir haben vor diesem Hintergrund daher an anderer Stelle formuliert:

„Porter and Kramer basically tell the old story of economic rationality as the one and only tool of smart management, with faith in innovation and growth, and they celebrate a capitalism that now needs to adjust a little bit. But there is little chance that an increasingly critical civil society will buy into such a story" (Beschorner 2013a: 111).

CSV, so unsere Befürchtung, läuft damit an gesellschaftlichen Erwartungen und Herausforderungen aus systematischen Gründen vorbei, weil gesellschaftliche Kontexte bei ausschließlicher Verwendung von Nutzenkategorien - im Grunde gar nicht verstanden werden (können).

In diesen Zusammenhang ist auch der Hinweis von Wieland und Heck (2013: 17) interessant, die anmerken, dass sich der Begriff „shared value“ auch in der neuen CSR-Definition der Europäischen Kommission (2011: 7) findet, hier gleichwohl in einer anderen Verwendung als bei Porter und Kramer:

„Während Porter/Kramer hauptsächlich die Unternehmen sowie die Gesellschaft als Ganze fokussieren, betont die Europäische Kommission die Beachtung der Interessen der Stakeholder von Unternehmen im Rahmen einer Integration von CSR in die strategische Unternehmensführung" (Wieland und Heck 2013: 17).

Wieland und Heck (2015) präzisieren in einem Briefwechsel mit den Autoren dieses Beitrages dazu:

„Die normativen Erwartungen von Politik und Gesellschaft müssen von den Unternehmen ernst genommen und adressiert werden, wenn sie langfristig am Markt existieren und Wettbewerbsvorteile generieren wollen. Stakeholder von Unternehmen sind nicht mehr nur externe Anspruchsteller, sondern konstruktive Elemente der täglichen Unternehmenspraxis, deren Interessen in die unternehmerischen Entscheidungen inkludiert werden sollen. Dieses normative Verständnis des ,shared value“ ist zu unterscheiden von einem „shared value“-Konzept bei Porter und Kramer, dessen Referenzpunkt die Erschließung von Marktchancen für ein Unternehmen ist. Letzteres kann ei- 
ne Konsequenz der normativen strategischen Ausrichtung eines Unternehmens sein, nicht aber die Engführung der Strategie auf Markterschließung selbst."

Das Problem des CSV-Ansatzes ist aber nicht nur die prominente Verwendung des Nutzenbegriffs, wie sie auch strategischen Stakeholderansätzen zugrunde liegt (vgl. z.B. Ulrich 1997: 438ff.; Waxenberger/Spence 2003), sondern auch das „framing“ des Verhältnisses von Unternehmen und Gesellschaft in weiten Teilen der (auch unternehmensethischen) Literatur insgesamt, die durch einen Stakeholderansatz per definitionem „unternehmenszentriert“ denkt: eine Betrachtungsweise von „relationships established between the focal organization and stakeholders" (Jonker/Foster 2002: 190); der Planet Unternehmen steht im Mittelpunkt, Stakeholder umkreisen ihn als Satelliten.

Wir haben an anderer Stelle (vgl. Beschorner 2004; 2013b) ausführlicher dargelegt, dass sich für eine theoretische wie empirische Bearbeitung einer Verhältnisbestimmung von Unternehmen und Gesellschaft die Notwendigkeit ergibt, eine Forschungsperspektive zu konzeptualisieren, die Akteurskonstellationen und -interaktionen untersucht. Weder die Unterscheidung zwischen konstitutioneller und nachkonstitutioneller Ebene (Spielregeln und Spielzüge) noch die Analyse von (bloß bilateralen) Stakeholderbeziehungen erscheinen uns dafür in hinreichender Weise geeignet (wenn auch in beiderlei Hinsicht anschlussfähig).

Damit verbunden ist ein weitreichender Perspektivenwechsel von einer ,gesellschaftsorientierten Unternehmenslehre zur unternehmensorientierten Gesellschaftslehre“ (Beschorner 2004), der den Begriff des „organisationalen Felds“ aus dem soziologischen Neo-Institutionalismus (vgl. grundlegend die Beiträge in: Powell/DiMaggio 1991/1983) bemüht. Eine „unternehmensorientierte Gesellschaftslehre“ tritt damit einerseits komplementär zum Planet-Satelliten-Modell eines Stakeholderansatzes. Sie ergänzt andererseits die Unterscheidung zwischen Spielregeln und Spielzügen, indem nicht mehr nur davon ausgegangen werden kann, dass die Spielregeln bloß extern gegeben, sondern, wie Edelman und Suchman (1997: 507) zeigen, selbst oft das Ergebnis von Kommunikations- und Aushandlungsprozessen zwischen regulierenden und zu regulierenden Organisationen darstellen.

Unternehmen (und andere Organisationen) treten in dieser Perspektive als aktive Mitgestalter der Spielregeln in Erscheinung, denen eine ordnungspolitische (Mit-) Verantwortung zukommt (vgl. dazu ausführlicher Beschorner 2004; Beschorner/Schank 2012). Über eine Betrachtung in organisationalen Feldern werden Unternehmen in der Gesellschaft verortet und damit als gesellschaftliche, governancepolitische Akteure überhaupt erst adressierbar.

\section{5. $\quad .$. und die Rolle der Wissenschaften}

Nein, bescheiden ist Herr Porter nicht, denn es sei schließlich CSV und nicht etwa CSR gewesen, das zwischenzeitlich zu ,substantial changes in behavior of corporations around the world“ (Porter/Kramer 2014: 149) geführt habe. CSV erfasse die Welt, wie sie wirklich sei: „it aligns social progress with corporate self interest in a concrete and highly tangible way, including with those 'old strategy models' that capture the reality of competition and prevailing corporate practice" (ebd.: 150). 
Ganz abgesehen von der empirischen Stichhaltigkeit einer solchen Behauptung wirft eine solche Formulierung über die (angebliche) „Praxis“ die Frage nach der Rolle und Verantwortung von Wissenschaftlern in diesem Diskurs mit der Praxis auf. Man kann Porter und Kramer zugutehalten, dass sie mit der Formulierung „Wertschöpfung“ statt „Verantwortung“ oder gar „Ethik“ nicht nur gutes (Selbst-)Marketing betreiben, sondern möglicherweise auch den richtigen Ton getroffen haben, um von „Praktikern“ gehört zu werden. Zugleich bekräftigen sie qua ihres Einflusses damit jedoch aktuelle Praktiken in der Wirtschaft, die sie zuvor als Gefahren für die Legitimität des Kapitalismus identifiziert haben. Porter und Kramer geben also einem System den wissenschaftlichen Segen, das sie aufgrund seiner bisherigen Funktionsweise als gefährdet ansehen.

Man kann nun wie Dyllick (2014) von einer grundsätzlichen „Ambiguität“ sprechen, die allen Wissenschaftlern, die sich mit wirtschaftsethischen Themen beschäftigen, anhaftet. So würde man in der Forschung und damit im Dialog mit Peers durchaus über die Grenzen von Konzepten wie CSV sprechen, während man in der Management-Fortbildung den Business Case, Reputationsgewinne, Wettbewerbsvorteile etc. in den Vordergrund stelle, um die Manager ,abzuholen“. Selbst wenn dies für einen großen Teil der Weiterbildung und auch der Lehre gelten sollte, so wirft es doch die Frage auf, warum der aktuelle Wissens- und Diskussionsstand nicht gelehrt werden sollte. Wird berufserfahrenen Professionals und Managern die Fähigkeit abgesprochen, sich mit Inhalten und Fragen zu beschäftigen, die nicht bereits Bekanntes bestätigen? Wählt man lieber den Weg des geringeren Widerstandes und übergeht essentielle Verantwortungsfragen zugunsten leicht verdaulicher Inhalte und eines gewissen Wohlfühlfaktors?

Es ist unbestritten, dass gerade die Weiterbildung eine Konkretisierung theoretischer Positionen und wissenschaftlicher Konzepte braucht, um einen praktischen Nutzen zu stiften. Es besteht indes ein großer Unterschied, ob man dafür über Instrumente und Verhaltensweisen spricht, mit denen Verantwortung implementiert werden kann, oder ob man ein intellektuelles Schlangenöl verkauft und damit den Menschen in Unternehmen ihre moralische Mündigkeit und Reflexionsfähigkeit abspricht.

Die Auseinandersetzung mit und Vermittlung von unternehmensethischen Fragen und Lösungsansätzen ist herausfordernd, sowohl für die Wissenschaft wie für die Praxis. Diesen Herausforderungen gilt es mit angemessenen Konzepten zu begegnen.

\section{Literaturverzeichnis}

Beschorner, T. (2004): Unternehmensethische Untersuchungen aus gesellschaftlicher Perspektive. Von der gesellschaftsorientierten Unternehmenslehre zur unternehmensorientierten Gesellschaftslehre, in: Zeitschrift für Wirtschafts- und Unternehmensethik, Jg. 5/Heft 3, 255-276.

Beschorner, T. (2013a): Creating Shared Value: The One-Trick Pony Approach - A Comment on Michael Porter and Mark Kramer, in: Business Ethics Journal Review, Vol. 1/No. 17, $106-112$. 
Beschorner, T. (2013b): Kulturalistische Wirtschaftsethik: Grundzüge einer Theorie der Anwendung, in: Zeitschrift für Wirtschafts- und Unternehmensethik, Jg. 14/Heft 3, 346372.

Beschorner, T./Hajduk, T. (2012): Vom ehrbaren Kaufmann zur Unternehmensverantwortung, in: Forum Wirtschaftsethik Online, Heft 2, 2-7.

Beschorner, T./Hajduk, T./Simeonov, S. (Hrsg.) (2013): Corporate Responsibility in Europe. Government Involvement in Sector-specific Initiatives, Gütersloh: Verlag Bertelsmann Stiftung.

Beschorner, T./Schank, C. (2012): CSR - zur Bürgerrolle und Verantwortung von Unternehmen, in: Schneider, A./Schmidpeter, R. (Hrsg.): Corporate Social Responsibility: Verantwortungsvolle Unternehmensführung in Theorie und Praxis, Berlin: Springer, 155-164.

Crane, A./Palazzo, G./Spence, L. J./Matten, D. (2014): Contesting the Value of the Shared Value Concept, in: California Management Review, Vol. 56/No. 2, 130-153.

Curbach, J. (2009): Die Corporate-Social-Responsibility-Bewegung, Wiesbaden: VS Verlag für Sozialwissenschaften.

Donaldson, T. (2014): Shared values that are lost in translation, in: Financial Times, 23.04.2014. Link: http://www.ft.com/intl/cms/s/2/bfdfff94-b34c-11e3-b09d-00144feabdc0.html\#axzz 3O9BYyPm5 (zuletzt abgerufen am 08.01.2015).

Dyllick, T. (2014): The opposing perspectives on creating shared value, in: Financial Times, 24.04.2014. Link: http://www.ft.com/intl/cms/s/2/88013970-b34d-11e3-b09d00144feabdc0.html\#axzz33cvuxOQI (zuletzt abgerufen am 08.01.2015).

Economist (2011): Oh, Mr Porter, in: The Economist, 10.03.2011. Link: http://www.econo mist.com/node/18330445 (zuletzt abgerufen am 8.1.2015).

Edelman, L. B./Suchman, M. C. (1997): The Legal Environments of Organizations, in: Annual Review of Sociology, Vol. 23/No. 1, 479-515.

Europäische Kommission (2011): Eine neue EU-Strategie (2011-14) für die soziale Verantwortung der Unternehmen (CSR). Link: http://ec.europa.eu/enterprise/newsroom/cf/_get document.cfm?doc_id=7008 (zuletzt abgerufen am 12.01.2015)

Friedman, M. (1970): A Friedman doctrine - The Social Responsibility of Business Is to Increase Its Profits, in: The New York Times Magazine, 13.9.1970, 32-33, 122-124.

Gelbman, U./Baumgartner, R. J. (2012): Strategische Implementierung von CSR in KMU, in: Schneider, A./Schmidpeter, R. (Hrsg.): Corporate Social Responsibility, Berlin: Springer, 285-298.

Hajduk, T. (2014): Stichwort: Ehrbarer Kaufmann, in: CSR NEWS. Link: http://csrnews.net/main/2014/03/23/ehrbarer-kaufmann/ (zuletzt abgerufen am 10.01.2015).

Hajduk, T. (2015): Bei Friedman nichts Neues. Anmerkungen zu einem falsch verstandenen Klassiker der CSR-Diskussion, in: Aufderheide, D./Dabrowski, M. (Hrsg.): Markt und Verantwortung. Wirtschaftsethische und moralökonomische Perspektiven, Berlin: Duncker \& Humblot, 89-98.

Hajduk, T./Schank, C. (2015): The Model of the Honourable Merchant: Bridging Compliance and Integrity?, in: Sison, A. (ed.): Handbook of Virtue Ethics in Business and Management, Berlin und Heidelberg: Springer (im Erscheinen).

Hartman, L. P./Werbane, P. H. (2013): Shared Value as an Incomplete Mental Model, in: Business Ethics Journal Review, Vol. 1/No. 6, 36-43. 
Jonker, J./Foster, D. (2002): Stakeholder Excellence? Framing the Evolution and Complexity of a Stakeholder Perspective of the Firm, in: Corporate Social Responsibility and Environmental Management, Vol. 9/No. 4, 187-195.

Lorentschitsch, B./Walker, T. (2012): Vom integrierten zum integrativen CSR-Managementansatz, in: Schneider, A./Schmidpeter, R. (Hrsg.): Corporate Social Responsibility, Berlin: Springer, 299-316.

Matten, D./Crane, A. (2005): Corporate Citizenship: Towards an Extented Theoretical Conceptualization, in: Academy of Management Review, Vol. 30/No. 1, 166-179.

Palazzo, G./Scherer, A. G. (2007): Toward a Political Conception of Corporate Responsibility: Business and Society Seen from a Habermasian Perspective, in: Academy of Management Review, Vol. 32/No. 4, 1096-1120.

Post, J. E./Preston, L. E./Sachs, S. (2012): Redefining the Corporation. Stakeholder Management and Organizational Wealth, Stanford: Stanford University Press.

Powell, W. W./DiMaggio, P. (Eds.) (1991/1983): The New Institutionalism in Organizational Analysis, Chicago, London: The University of Chicago Press.

Porter, M. E./Kramer, M. R. (2011): Creating Shared Value. How to Reinvent Capitalism - and Unleash a Wave of Innovation and Growth, in: Harvard Business Review, Vol. 89/No. 1, 62-77.

Porter, M. E./Kramer, M. R. (2014): A Response to Andrew Crane's et al.'s article, in: California Management Review, Vol. 56/No. 2, 149-151.

Preston, L. E. (1975): Corporation and Society: The Search for a Paradigm, in: Journal of Economic Literature, Vol. 13/No. 2, 434-53.

Schmiedeknecht, M. H./Wieland J. (2012): ISO 26000, 7 Grundsätze, 6 Kernthemen, in: Schneider, A./Schmidpeter, R. (Hrsg.): Corporate Social Responsibility, Berlin: Springer, 259-270.

Scholy, M. (2014): Stichworte: Creating Shared Value, Shared Value, in: CSR NEWS. Link: http://csr-news.net/main/2014/03/23/creating-shared-value-2/ (zuletzt abgerufen am 10.01.2015).

Waxenberger, B./Spence, L. J. (2003): Reinterpretation of a Metaphor: from Stakes to Claims, in: Strategic Change, Vol. 12/No. 5, 239-249.

Wieland, J. (1999): Die Ethik der Governance, Marburg: Metropolis.

Wieland, J./Heck A. E. H. (2013): Shared Value durch Stakeholder Governance, Marburg: Metropolis.

Wieland, J./Heck A. E. H. (2015): Creating Shared Value: E-Mail-Korrespondenz mit Thomas Beschorner und Thomas Hajduk am 7. Januar 2015.

Wolf, K. D./Schwindenhammer, S. (2011): Vom Business Case zum Public Case? Der Beitrag privater Selbstregulierung zu Global Governance, in: Zeitschrift für Wirtschafts- und Unternehmensethik, Jg. 12/Heft 1, 10-28.

Ulrich, P. (1997): Integrative Wirtschaftsethik. Grundlagen einer lebensdienlichen Ökonomie. Bern, Stuttgart, Wien: Haupt Verlag.

Zadek, S. (2001): The Civil Corporation: The New Economy of Corporate Citizenship, London: Routledge. 